\title{
Rapid and sensitive detection of small biomolecule by capacitive sensing and low field AC electrothermal effect
}

\author{
Haochen Cui ${ }^{1}$, Cheng Cheng ${ }^{1}$, Xiaogang Lin ${ }^{1,2}$, Jayne $\mathrm{Wu}^{1,}{ }^{*}$, Jiangang Chen ${ }^{3}$, Shigetoshi Eda ${ }^{4}$, \\ and Quan Yuan ${ }^{1}$ \\ 1. Department of Electrical Engineering and Computer Science, the University of Tennessee, Knoxville, \\ TN 37996, USA \\ 2. Key Laboratory of Optoelectronic Technology and System of the Education Ministry of China, \\ Chongqing University, Chongqing 400044, China \\ 3. Department of Public Health, the University of Tennessee, Knoxville, TN 37996, USA \\ 4. Department of Forestry, Wildlife and Fisheries, The University of Tennessee, Knoxville, TN 37996, \\ USA \\ *: corresponding author, jwu10@utk.edu
}

\begin{abstract}
Conventional affinity biosensor typically relies on passive diffusion of analytes for binding reaction, which in many cases leads to long response time and lack of sensitivity. Recent research showed that directed particle motion towards sensor electrodes could be induced in sample matrix by applying an inhomogeneous AC electric field, often with AC dielectrophoresis as the responsible mechanism. As a result, shorter assay time and higher sensitivity can be achieved. Previously, we demonstrated a rapid and sensitive AC capacitive affinity sensor, which integrates low voltage AC dielectrophoresis into label-free capacitive measurement to achieve a single-step operation without any wash steps for clinical samples. However, dielectrophoretic force is rather short-ranged, and is also proportional to the size of target biomolecules/particles. Therefore, to detect target molecule at diluted concentrations or small molecule, improvement in sensitivity by dielectrophoresis could be quite limited. Alternatively, AC electric field can also produce microfluidic movement to carry biomolecules to sensors, which is of long range and size independent. This work demonstrates the use of low voltage AC electrothermal effect to enhance and accelerate the detection of low abundance and small target molecules by AC capacitive sensing with simultaneous AC electrokinetic enrichment. Electrode designs were studied for their effectiveness in AC electrothermal capacitive sensing. Electrodes with larger characteristic length were found to be more amenable to inducing AC electrothermal convection and were successfully used to detect low abundance protein and femto-molar level small molecules.
\end{abstract}

Key words: AC electrokinetics; affinity sensor; capacitive biosensor; point of care diagnosis. 


\section{Introduction}

Point-of-care diagnostics is becoming increasingly important for improving world health, and biosensors have shown great potential in realizing rapid, inexpensive and portable on-site diagnosis [1, 2]. Among various types of biosensors, biosensors based on electrical signal, such as electrochemical sensors and impedimetric biosensors, have attracted great interest due to their ability to achieve high sensitivity detection with relatively simple instrumentation and operation [3-6]. The most commercially successful biosensor is glucose meter based on enzymatic redox reaction. However, on-site detection of low abundance analyte is much less successful, especially for affinity based detection. Affinity sensors detect biological particles based on specific binding between analyte and probe molecules [7-9]. Conventional affinity biosensors, including microarrays [10-12] and enzyme-linked immunosorbent assays (ELISA) [13], require long incubation time and sophisticated instrumentation for field use. So a low cost, portable and high sensitive affinity sensor is highly desired. Electrical based detection will provide a low cost solution to detection instrumentation. Here, our work will show that an electrical sensing method, AC electrokinetics-based capacitive sensing, can also speed up detection and improve sensitivity for affinity sensors, in addition to low cost and portability.

Affinity biosensors rely on the binding of analytes with receptor probes to achieve detection, so the meeting of probe and analyte molecules is crucial. By passive molecular diffusion, detection often requires long incubation time, ranging from minutes to several hours [14], especially for low abundance analyte. Many biomarkers and analytes associated with diseases and pollution exist in biological fluids and environments with low abundance, typically in the range of $\mathrm{fg} / \mathrm{mL}$ to $\mathrm{ng} / \mathrm{mL}$ [15], which is highly challenging to detect. To achieve usable sensitivity, enzymatic [16] florescent [17] or particle label [18, 19] is often required for signal amplification, which significantly complicates the detection procedure. For label-free detection, to overcome the diffusion barrier, some research groups employed external stirring to speed up macromolecule transport [20-22], including alternating current electrokinetics (ACEK) method. ACEK [23], implemented by microelectrodes immersed in sample fluids, induces directional particle or fluid motion by externally applied AC electric fields over the electrodes. When applying inhomogeneous AC electric field to biological samples, possible ACEK effects that can be induced around microelectrodes include DEP and AC electrothermal effects. DEP force is directly applied over particles, and is proportional to the size of target biomolecules/particles. So enrichment by DEP is more effective with larger biomolecules. DEP is also rather short-ranged, with its effective range typically within microns. Besides DEP, AC electric field can also produce rotating vortex-like microfluidic movement above electrode surface, which is known as AC electrothermal (ACET) effect [24, 25]. As microflows can carry target biomolecules to the electrodes for binding, analyte enrichment by ACET effect is of long range and size independent.

Since its advent in 1990s, ACEK has been studied and utilized by many researchers to accelerate the movement of macromolecules towards sensing areas [21, 22, 26, 27], and 
improvement in sensor responses has been observed repeatedly when ACEK effects are incorporated. However, in almost all the existing ACEK devices, the amplitude of AC signal used is usually around $10 \mathrm{~V}[21,28,29]$, and the ACEK preconcentration step is separate from the detection step [26]. Applying higher than 1V voltage over biofluids raises the concerns of electrolysis [30], biofouling [31], etc., and an additional preconcentration step adds to the operation complexity. These reasons severely limit the use of ACEK in biosensing. While there was one report using $100 \mathrm{mV}$ AC to enhance binding assay, the target protein were tagged with fluorescent microparticles for signal amplification, and the detection had to be endpoint detection due to the separate preconcentration/incubation step [32].

Recently, we developed a rapid and simple to operate affinity sensing technique, known as "ACEK capacitive sensing method" [33]. This method integrates low voltage $(<1 \mathrm{~V})$ dielectrophoresis (DEP) into label-free capacitive measurement to achieve a single-step operation without any wash steps for clinical samples [33, 34]. With interdigitated microelectrodes as the sensor, the interfacial capacitance of electrodes/sample solution is monitored at a fixed frequency AC signal between $10 \mathrm{mV}-500 \mathrm{mV}$. Specific binding at electrode surface is detectable through a change in the interfacial capacitance [35]. Capacitive sensing is usually done at low voltage such as $5 \mathrm{mV}$. Here the capacitance change is measured at a higher AC voltage, which will also induce ACEK effects simultaneously during measurement in the fluid around the electrodes. The whole detection process is a one-step operation that completes at the end of capacitance measurement. Since the AC signal will accelerate the transport of analyte towards the electrodes for binding, the response time is usually less than one minute. So this method is much simpler and faster than commonly used ELISA procedure requiring multiplewash/ hour-long incubation [36]. While no particle movement can be visualized for ACEK effects due to the use of low voltage, ACEK capacitive sensing exhibits significant improvement in response time and detection sensitivity. Our prior work successfully demonstrated detection of specific protein and virus particles from clinical samples (serum and nasal swab) with high sensitivity and specificity and fast response time $[33,34]$.

In the prior work detecting protein or larger bioparticles, DEP was determined to be the dominant ACEK mechanism of analyte enrichment, since the response magnitude showed a bellshape dependence on AC frequency. As DEP force is size dependent and short-ranged, when detecting target molecule at diluted concentration or small molecule, improvement by DEP becomes limited [37]. To further understand and improve ACEK capacitive sensing method, this work investigated the feasibility of inducing low voltage AC electrothermal (ACET) flows to enhance the detection of diluted and/or small molecules target. Different electrode patterns are studied and compared for their effectiveness in capacitive detection of antigen-antibody binding and aptamer-small molecule binding. Using an electrode design of choice, improved results have been achieved for low abundance protein and small molecule, reaching detection limits of 1 $\mathrm{ng} / \mathrm{mL}$ for protein (IgG) and $10 \mathrm{fM}$ for small molecule (bisphenol-A) within one minute. 


\section{Theory}

When electrodes are immersed in an aqueous solution, electric double layer (EDL) will form at the interface of electrodes and solution. EDL can be electrically regarded as a capacitor, which is also known as the interfacial capacitance $\left(\boldsymbol{C}_{\boldsymbol{i n t}}\right)$ of the electrolytic cell. Past work has shown that $\boldsymbol{C}_{\boldsymbol{i n t}}$ can reflect with high sensitivity any physical changes (i.e. binding) at the electrode surfaces. The effect of interfering constituents in the test fluids mostly shows up through the fluid resistance, which is easily eliminated in capacitive sensing since only $\boldsymbol{C}_{\boldsymbol{i n t}}$ is measured. Detection of $\boldsymbol{C}_{\boldsymbol{i n t}}$ also minimizes problems with un-desired charge transfer pathways through surface in a complex fluid that troubles enzymatic sensing. Consequently, no wash step is required for ACEK capacitive sensing method, yet high selectivity can still be achieved. In this method, by using a fixed frequency of choice $(100 \mathrm{kHz})$ along with the dilution buffer we chose, the capacitive component of the measured impedance corresponds directly to the interfacial capacitance $\left(\boldsymbol{C}_{\text {int }}\right)$ of microelectrodes. So biomolecular binding at the sensor surface can be found quickly and directly without resorting to complicated data processing. Additionally, at this frequency, most of electric field drops over the fluid to induce ACEK effects such as DEP and ACET flows. Only a small portion of electric potential is applied between the electrode and fluid interface, so there is no appreciable effect on biomolecular attachment on the electrodes.

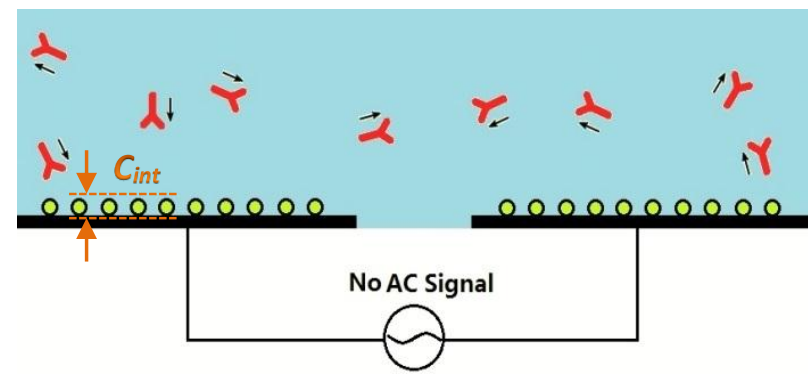

(a)

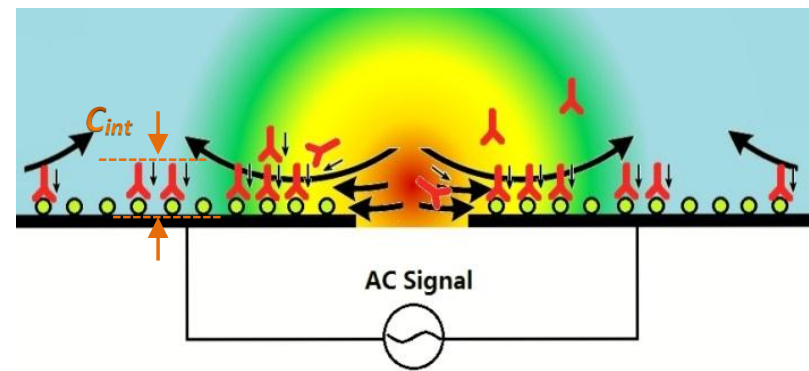

(b)

Figure.1 Process of specific analyte (antibody) binds to the probe molecules (antigen) immobilized on the electrode for detection. (a) Random movement of analyte by thermal diffusion; and (b) directed movement of analyte towards the electrodes for binding when aided by DEP and AC electrothermal effects. The color shows temperature distribution in the fluid, with the temperature maximum between the electrodes. $C_{i n t}$ becomes lower when more biomacromolecules are attached to the electrode surface.

In this ACEK capacitive method, when reading capacitance at a pre-determined AC frequency and voltage, an inhomogeneous AC electric field is applied through microelectrodes to an aqueous solution, and ACEK effects take place. Consequently, particle and fluid movement 
could be induced around the microelectrodes to achieve in-situ enrichment of macromolecules. Directional particle movement can be induced by DEP, and can also be caused by microflow convection such as AC electroosmosis (ACEO) [38, 39] or AC electrothermal (ACET) flow [25, $40]$ to reach the microelectrodes, as schematically shown in Figure.1.

DEP force on a spherical particle can be expressed as [41]:

$$
\mathrm{F}_{\mathrm{DEP}}=\pi \mathrm{a}^{3} \varepsilon_{\mathrm{m}} \operatorname{Re}\left[\frac{\varepsilon_{\mathrm{p}}^{*}-\varepsilon_{\mathrm{m}}^{*}}{\varepsilon_{\mathrm{p}}^{*}+2 \varepsilon_{\mathrm{m}}^{*}}\right] \nabla|\mathrm{E}|^{2} \approx \pi \mathrm{a}^{3} \varepsilon_{\mathrm{m}} \operatorname{Re}\left[\frac{\varepsilon_{\mathrm{p}}^{*}-\varepsilon_{\mathrm{m}}^{*}}{\varepsilon_{\mathrm{p}}^{*}+2 \varepsilon_{\mathrm{m}}^{*}}\right] \frac{|\mathrm{E}|^{2}}{r}
$$

where $\varepsilon^{*}=\varepsilon-j \sigma / \omega$ is the complex permittivity with $\varepsilon, \sigma$ and $\omega$ being permittivity, electrical conductivity and angular frequency; subscript $p$ and $m$ denote particle and medium, respectively. Re is the real part of a number, and $\left[\frac{\varepsilon_{\mathrm{p}}^{*}-\varepsilon_{\mathrm{m}}^{*}}{\varepsilon_{\mathrm{p}}^{*}+2 \varepsilon_{\mathrm{m}}^{*}}\right]$ is known as Clausius-Mossotti factor and is frequency dependent with a value between -0.5 and 1 . $a$ is the particle radius; $E$ is the electric field strength, and $r$ is half of the electrode gap. Based on eq. 1, for nanoscale macromolecules such as antibodies (small $a$ ), DEP is effective only with high $\nabla|E|^{2}$, i.e. high electric field gradient, which means that macromolecules need to be within a very short distance from the electrodes to experience sufficient DEP attraction.

If biomolecules that are some distance away from the electrode surface can still be routed towards the electrodes, then lower limit of detection can be achieved. For this reason, it is advantageous to utilize fluid movement for long range/low abundance detection. Microflows can be generated by AC electric field through two ACEK flow mechanisms known as ACEO and ACET effect, which can assist in the enrichment of macromolecules by carrying macromolecules to the electrodes for binding. ACEO phenomena typically occur at low ionic strength [42], and ACEO flow velocity has been observed to decrease significantly with increasing fluid's electrical conductivity and eventually drop to zero at above $0.085 \mathrm{~S} / \mathrm{m}$ [43], making it unsuitable for most bioassays. This work uses $15 \mathrm{mM} \mathrm{Na}+$ phosphate buffered saline (PBS) with $\sigma=\sim 0.18 \mathrm{~S} / \mathrm{m}$, so ACEO effect will be negligible. In comparison, ACET effect becomes increasingly pronounced with higher fluid conductivity, since higher temperature gradient could be generated, leading to stronger ACET flows. ACET flows are induced by inhomogeneous electric field and the resultant temperature rise, which further results in localized changes in the electric conductivity and permittivity of the fluid. To maintain electrical neutrality, electrical charges are induced in the fluid, which move under the influence of the electric field to produce microflows. With planar electrodes, ACET effect will induce vortices above each electrode, and the microflows will convect the embedded particles towards the electrode surface [44, 45]. Below a few $\mathrm{MHz}$, ACET effect only depends on AC voltage level. There is no dependence on the size of 
bioparticles or AC frequency, making ACET effect suitable for enrichment of a broad range of bioparticles. ACET force $\left(\mathrm{F}_{\mathrm{ET}}\right)$ on fluid can be expressed as [41]:

$$
F_{E T}=0.011 \frac{\varepsilon \sigma}{k} \pi r E^{4},
$$

for which $300 \mathrm{~K}$ room temperature is assumed. $k$ in Eq. (2) is the thermal conductivity of sample fluid. In Eqs. (1) and (2), electric field strength $E$ is approximated by electric potential $V$ over the half circle over electrode gap, i.e. $\mathrm{E}=\mathrm{V} / \pi \mathrm{r}$ ), and root mean square values are used for electric field.

Based on Eqs. (1) and (2), two differences between DEP and ACET forces can be observed. First, ACET force increases with electric field strength $E$ more strongly than DEP forces, as DEP force $F_{D E P} \propto|E|^{2}$ and ACET force $F_{A C E T} \propto|E|^{4}$. Second, for a given electric field strength, DEP effect weakens with electrode gap while ACET effect becomes stronger with electrode gap. The relative importance of DEP and ACET effect is clearly dependent on electrode design, which is supported by numerical modelling of ACEK induction by microelectrodes [27]. In the previous work on ACEK capacitive sensors [33], microelectrodes modified from off-the-shelf surface acoustic wave (SAW) resonators were adopted as the sensing element. In that work, frequency dependency in the sensor response was clearly observed, indicating that analyte enrichment, to a large degree, was caused by DEP. It was hypothesized that the sensor performance could be further improved if ACET microflows could be adequately exploited to assist biomolecular transport. So here, ACEK biosensing by larger electrodes will be studied to see if ACET flow can be adequately induced, still at low electric field strength, to enhance binding. The benefits of ACET enrichment include a longer effective range, i.e. lower limit of detection, and being effective with small molecules since ACET flow is size-independent. Our prior work [27, 46] demonstrated that $5 \mu \mathrm{m}$ electrodes was effective at inducing ACET microflows for biofluid convection at several volts, and the same electrode designed will be used here and compared with past sensor performance.

\section{Devices and methods}

\subsection{Microelectrode sensors}

To compare different sensing electrodes, this work used planar microelectrodes fabricated in house on silicon wafers and modified SAW resonators, as shown in Fig.2. In-house fabricated microelectrode arrays have interdigitated configuration with two different dimensions, electrodes with a characteristic length of $2 \mu \mathrm{m}$ (with widths of $2 \mu \mathrm{m}$ separated by $2 \mu \mathrm{m}$ gaps), denoted as 2 $\mu \mathrm{m}$ electrodes, and electrodes with a characteristic length of $5 \mu \mathrm{m}$ (with widths of $5 \mu \mathrm{m}$ and 25 
$\mu \mathrm{m}$ separated by $5 \mu \mathrm{m}$ and $25 \mu \mathrm{m}$ gaps), denoted as $5 \mu \mathrm{m}$ electrodes. A silicone microchamber (2 $\mathrm{mm}$ in diameter and $0.9 \mathrm{~mm}$ in depth, JTR24R, Grace ${ }^{\mathrm{TM}}$ Bio-labs) was sealed onto the wafer with electrodes inside for sample loading. In addition, commercial SAW resonators were modified to expose the interdigitated electrodes inside for use, which has a characteristic length of $1.5 \mu \mathrm{m}$. (Zoomed-in images of microelectrodes can be found in Supplementary Material section.) As shown in Fig.2b, the electrode array was about $1 \mathrm{~mm}$ in total length and $0.7 \mathrm{~mm}$ in width placed on a ceramic support in the middle of SAW chip package. The metal casing around the electrode chip is about $4 \mathrm{~mm}(\mathrm{~L}) \times 2.5 \mathrm{~mm}(\mathrm{~W}) \times 1 \mathrm{~mm}(\mathrm{H})=10 \mu \mathrm{L}$, serving as a microchamber for sample solutions. The microchambers in both cases are sufficiently high, so that electric fields and ACEK effects can fully develop, as shown by numerical simulation [47].

Before being functionalized as affinity sensors, the microelectrodes were thoroughly cleaned. The electrodes were first soaked in acetone for 10 minutes, then rinsed by isopropyl alcohol for 15 seconds, deionized water for another 15 seconds, and finally dried with air gun. The quality of the prepared electrodes was evaluated by measuring the interfacial capacitance, as described in Ref. [37].

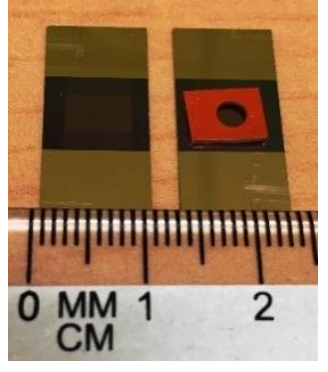

(a)

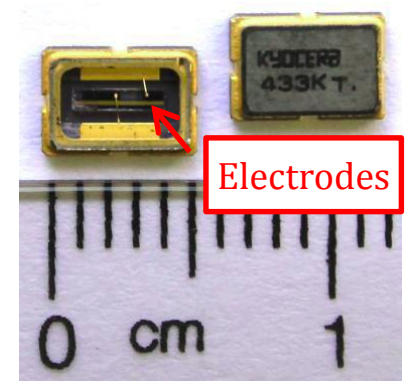

(b)

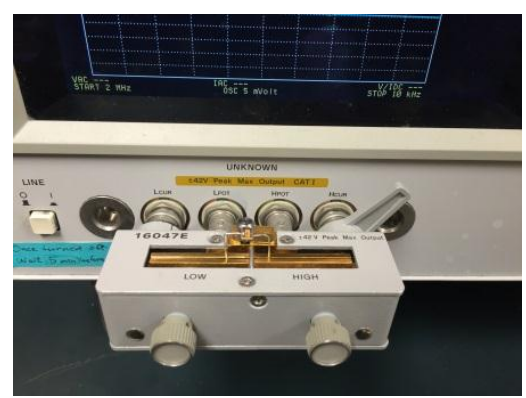

(c)

Figure.2 (a) An array of planar microelectrodes on silicon wafer with silicon microchamber over it; (b) modified SAW resonators showing the microelectrodes inside. The bright line in the middle of chip is the microelectrode array; and (c) a SAW resonator electrode-sensor under test.

\subsection{Sample preparation and electrode functionalization}

Sample solutions were prepared using $1 \mathrm{mM}$ phosphate buffer $(\mathrm{pH} 7.0)$ containing $15 \mathrm{mM}$ $\mathrm{NaCl}$, referred to as 0.1x phosphate-buffered saline (PBS). 0.1x PBS-T is used for rinsing, which is $0.1 \mathrm{x}$ PBS containing $0.05 \mathrm{v} / \mathrm{v} \%$ Tween 20 (Fisher Scientific, Pittsburgh, PA).

Before binding assay, the electrode surface was functionalized by immobilizing probe molecules and blocking. For protein sensors, bovine IgG whole molecules (Jackson 
ImmunoResearch Laboratories Inc., West Grove, PA) were used as the probe molecules, which was diluted to a concentration of $10 \mu \mathrm{g} / \mathrm{mL}$ using $0.1 \mathrm{x}$ PBS. The bovine IgG solution was loaded into the microchamber and incubated in a humidor at room temperature for 5 hours. Passive adsorption was used without any linker molecules. Afterwards, protein chips were blocked with 0.1x Buffer B, which is 0.1x PBS-T containing $10 \mathrm{v} / \mathrm{v} \%$ SuperBlock (PIERCE Biotechnology, Rockford, IL). A goat anti-bovine $\operatorname{IgG}(\mathrm{H}+\mathrm{L})$ antibody (unlabeled, Jackson ImmunoResearch Laboratories Inc.) stock solution with a concentration of $2.4 \mathrm{mg} / \mathrm{mL}$ was used to prepare anti-bovine IgG antibody sample solutions. The samples were 1:10 serial diluted to concentrations of 100,10 , and $1 \mathrm{ng} / \mathrm{mL}$ by serial dilutions. $0.1 \mathrm{X}$ PBS was used as the sample dilutant and also as the control sample.

For small molecule detection experiments, aptamer specific to bisphenol A (BPA) [48]' with 5' thiol modifier C6 SH (Sangon Biotech Co., Ltd, Shanghai, China) was immobilized onto the electrodes at a concentration of $1 \mu \mathrm{M}$, which was also incubated in a humidor at room temperature for 5 hours. The sequence of the aptamer ( 5 ' to 3') is 5' -CCG GTG GGT GGTCAG GTG GGATAG CGT TCC GCG TAT GGC CCA GCG CAT CAC GGG TTC GCA CCA-3’. Then the aptamer electrodes were blocked with Triton X-100 (0.1v/v \%), which was prepared by adding $10 \mu \mathrm{L}$ of Triton X-100 solution in $10 \mathrm{~mL}$ of distilled water [49]. For the detection of small molecule, BPA molecule was 1:10 serial diluted in 0.1x PBS with concentration ranging from 1 to 1000 fM. 4, 4'-Isopropylidenediphenol (BPA) and dimethyl Sulfoxide (DMSO), $\geq 99.7 \%$ were purchased from Fisher Scientific, Pittsburgh, PA. $11.3 \mathrm{mg}$ BPA was dissolved in $5 \mathrm{~mL}$ DMSO to make $10 \mathrm{mM}$ BPA stock solution. Serial 1:10 dilutions were then made with DMSO from stock solution to make standard concentrations of $1 \mu \mathrm{M}, 100 \mathrm{nM}, 10 \mathrm{nM}$, and $1 \mathrm{nM}$. The above concentrations were further diluted 1:1,000,000 in 0.1X PBS to make the working solution of $1 \mathrm{pM}, 100 \mathrm{fM}, 10 \mathrm{fM}$, and $1 \mathrm{fM}$ for testing. The concentrations of DMSO were kept constant in all the standards.

Non-specific binding experiments were also conducted to evaluate the specificity of this sensing method. Dummy electrodes were prepared by blocking the electrode surfaces without immobilizing probe molecules. Those electrodes were then used against sample solutions as described above. For small molecule BPA detection, in addition to dummy electrodes, bisphenol-S (BPS) was also tested against electrodes functionalized with BPA-aptamer. BPS is very similar in structure to BPA, in which the dimethylmethylene group $\left(\mathrm{C}\left(\mathrm{CH}_{3}\right)_{2}\right)$ is replaced with a sulfone group $\left(\mathrm{SO}_{2}\right)$. Different concentrations of BPS solutions were prepared using the same procedure for BPA solutions as described above.

\subsection{Measurement instrumentation}

The interfacial capacitance was continuously measured at $100 \mathrm{kHz}$ by an impedance analyzer (Agilent 4294A) for 30 seconds. The impedance analyzer will read the sensor impedance in the 
format of $R_{S}+j \frac{1}{\omega C_{S}}$, where $R_{S}$ and $C_{S}$ are the resistance and capacitance of the sensor when it is represented as a serial connection of a resistor and a capacitor. Here, $C_{S}$ is approximately half of interfacial capacitance. Such a treatment of sensor impedance was justified in our prior work [50]. Further, ACEK capacitive sensing method adopts a dimensionless quantity - the percentage change in the measured capacitance to indicate analyte deposition onto the electrode, which is the slope of normalized capacitance change versus time (\%/minute) and found by least square linear fitting method. Normalized capacitance is calculated as

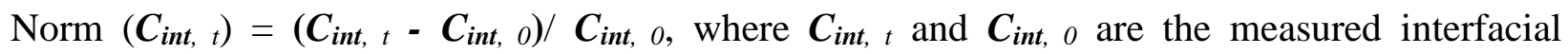
capacitances at time $\mathrm{t}$ and time zero, respectively. Doing so circumvents the need for a reference sensor, minimizes size difference from sensor to senor, further simplifying the detection procedure and instrumentation.

The impedance of $2 \mu \mathrm{m}$ and $5 \mu \mathrm{m}$ electrodes after loading sample is measured to be in the range of 70-100 $\Omega$ at $100 \mathrm{kHz}$, and the impedance analyzer used for capacitance measurement has an internal resistance of $25 \Omega$. Thus, loading effect cannot be neglected, i.e. the output voltage from the impedance analyzer is divided between the sensor and analyzer, and the actual voltage applied onto the sensor is lower than the nominal voltage set for the impedance analyzer. To obtain the actual voltage drop, an oscilloscope (Agilent DSO3062A) was connected to the sensor during measurement. The measured voltages showed around 30\% decrease from the nominal measuring voltage for $2 \mu \mathrm{m}$ and $5 \mu \mathrm{m}$ electrodes. Table. 1 gives the actual voltage drops corresponding to the displayed voltages on the impedance analyzer. The impedance of SAW chip electrodes is over $1 \mathrm{k} \Omega$, so the loading effect is negligible.

Table. 1 Measured voltage drop on $2 \mu \mathrm{m}$ and $5 \mu \mathrm{m}$ electrodes

(Unit: mVrms, rms: root mean square)

\begin{tabular}{|c|c|c|c|c|c|c|c|c|}
\hline Nominal voltage & 50 & 100 & 125 & 200 & 250 & 400 & 500 & 1000 \\
\hline Actual voltage & 41 & 78 & 89 & 140 & 181 & 272 & 335 & 650 \\
\hline
\end{tabular}

\section{Experiments and discussions}

\section{a) Protein detection}

First, to demonstrate the effect of electrode designs on protein enrichment and detection, sensor responses from three types of electrodes were obtained under similar electric field strength for a range of protein concentrations. Three types of sensing electrodes were used, SAW electrodes (i.e. $1.5 \mu \mathrm{m}$ electrodes), $2 \mu \mathrm{m}$ electrodes and $5 \mu \mathrm{m}$ electrodes on silicon wafers. All were functionalized with anti-IgG antigen. The characteristic electric field strength for a sensor was estimated as the applied voltage divided by the half circle over narrow electrode gap $(\mathrm{E}=$ $V / \pi r, \mathrm{r}$ is half of the electrode gap). So the electric field strengths were $42.4 \mathrm{mV} / \mu \mathrm{m}$ for SAW electrodes, $44.6 \mathrm{mV} / \mu \mathrm{m}$ for $2 \mu \mathrm{m}$ electrodes, and $42.7 \mathrm{mV} / \mu \mathrm{m}$ for $5 \mu \mathrm{m}$ electrodes. Figure. 3 gave 
the responses of the three different electrodes to various concentrations of IgG samples. To account for possible artifacts from applying electric signal to biological samples, buffer solution without analyte was tested on functionalized electrodes as control experiments. For non-specific binding tests, sample solution containing $10 \mathrm{ng} / \mathrm{mL}$ antibody for protein sensing were tested. Measurement at each data point was repeated three times.

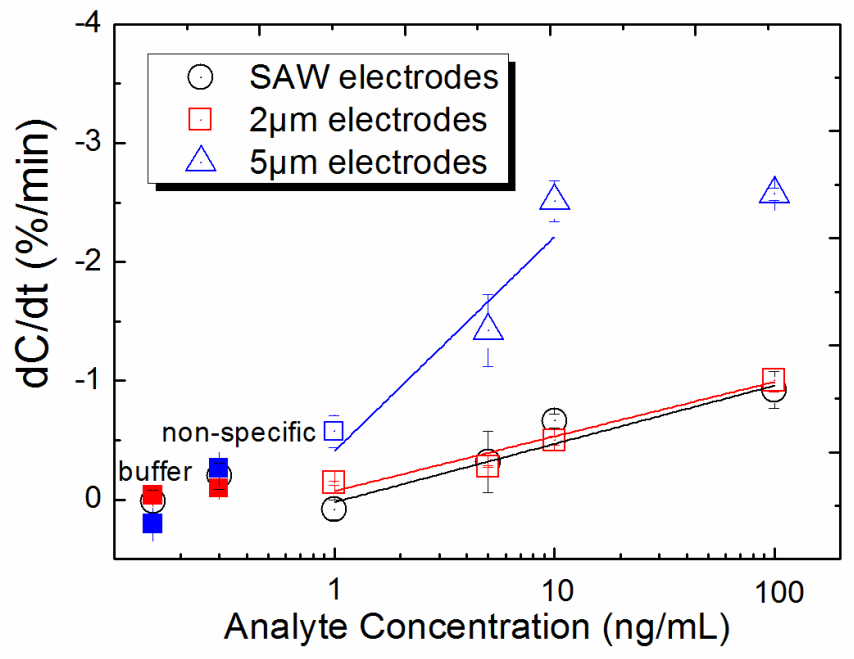

Figure.3 Binding responses from three different electrodes under similar electric field strength. Each test was repeated three times. Error bar indicates the standard deviation. Control results included both buffer solution and non-specific binding on un-functionalized electrodes.

In Fig.3, the control experiments using blank buffer exhibited very little responses. SAW electrodes, $2 \mu \mathrm{m}$ electrodes and $5 \mu \mathrm{m}$ electrodes showed capacitance change rates of $0.01 \pm$ $0.09 \% / \mathrm{min},-0.03 \pm 0.03 \% / \mathrm{min}$ and $0.21 \pm 0.05 \% / \mathrm{min}$, respectively. The outputs of nonspecific binding tests on un-functionalized electrodes were a little larger, but still rather low, $-0.19 \pm 0.10 \% / \mathrm{min}$ for SAW electrodes, $-0.09 \pm 0.03 \% / \mathrm{min}$ for $2 \mu \mathrm{m}$ electrodes and $-0.26 \pm$ $0.01 \% / \mathrm{min}$ for $5 \mu \mathrm{m}$ electrodes, attesting to the specificity of this sensing method. Also, based on Fig.3, sensor output more positive than $-0.30 \% / \mathrm{min}$ was defined as being non-reactive, i.e. negative response.

As could be seen, the capacitance change rates were very similar between SAW electrodes and $2 \mu \mathrm{m}$ electrodes for all the concentrations tested $(1,5,10$ and $100 \mathrm{ng} / \mathrm{mL})$, while $5 \mu \mathrm{m}$ electrodes exhibited much larger responses. The SAW and $2 \mu \mathrm{m}$ electrodes had a logarithmic dependence on IgG concentration ranging from 1 to $100 \mathrm{ng} / \mathrm{mL}$. Their sensitivities were found by linear curve fitting to be $-0.59 \pm 0.12$ and $-0.49 \pm 0.07 \% / \mathrm{log}(\mathrm{ng} / \mathrm{mL})$ with a Pearson correlation coefficient[51] $\mathrm{R}^{2}$ of 0.88 and 0.94 for SAW and $2 \mu \mathrm{m}$ electrodes, respectively. In comparison, $5 \mu \mathrm{m}$ electrodes yielded a sensitivity of $-1.80 \pm 0.69 \% / \log (\mathrm{ng} / \mathrm{mL})$, more than three times that 
of SAW and $2 \mu \mathrm{m}$ electrodes. The $5 \mu \mathrm{m}$ electrodes exhibited logarithmic dependence on IgG concentration from 1 to $10 \mathrm{ng} / \mathrm{mL}$. However, there was no increase in sensor response when the IgG level became higher than $10 \mathrm{ng} / \mathrm{mL}$, probably due to saturation of binding sites by strong ACEK enrichment.

With the limit of detection (LOD) being defined as more than three standard deviations from that of control tests, the LODs of SAW and $2 \mu \mathrm{m}$ electrodes were found to be $10 \mathrm{ng} / \mathrm{mL}$ (their capacitance change rates at $10 \mathrm{ng} / \mathrm{mL}$ were around $-0.50 \pm 0.04 \% / \mathrm{min})$. In contrast, $5 \mu \mathrm{m}$ electrodes showed a change rate of $-0.60 \pm 0.13 \% / \mathrm{min}$ for $1 \mathrm{ng} / \mathrm{mL} \mathrm{IgG} \mathrm{sample.} \mathrm{So} \mathrm{the} \mathrm{LOD}$ for $5 \mu \mathrm{m}$ electrodes was $1 \mathrm{ng} / \mathrm{mL}$, increasing by approximately one order of magnitude from SAW and $2 \mu \mathrm{m}$ electrodes.

Since the electric field strengths are similar between the electrodes, so the differences between their output magnitudes can only be attributed to the electrode geometric differences. Given the similar electrode characteristic dimensions between SAW and $2 \mu$ m electrodes, their responses were similar as expected. As an aside, it should also be noted that SAW and $2 \mu \mathrm{m}$ electrodes did have very different total electrode areas exposed to fluid. SAW electrode array had a total active area of $0.7 \mathrm{~mm}^{2}$, while $2 \mu \mathrm{m}$ electrode array has an active area of $\sim 4.9 \mathrm{~mm}^{2}$. The results showed that by using normalized capacitance change, the difference in total electrode areas and microchamber geometries will not have appreciable effects on the sensor response, increasing the test repeatability from device to device. The difference in sensor responses appeared to arise from their characteristic lengths. According to Eqs. (1) and (2), DEP is more pronounced with smaller electrodes, while ACET effect favors larger microelectrodes. As there was significant improvement in the sensitivity and LOD of $5 \mu \mathrm{m}$ electrodes over SAW and $2 \mu \mathrm{m}$ electrodes, it could be concluded that ACET effect will dominate for larger electrodes. 

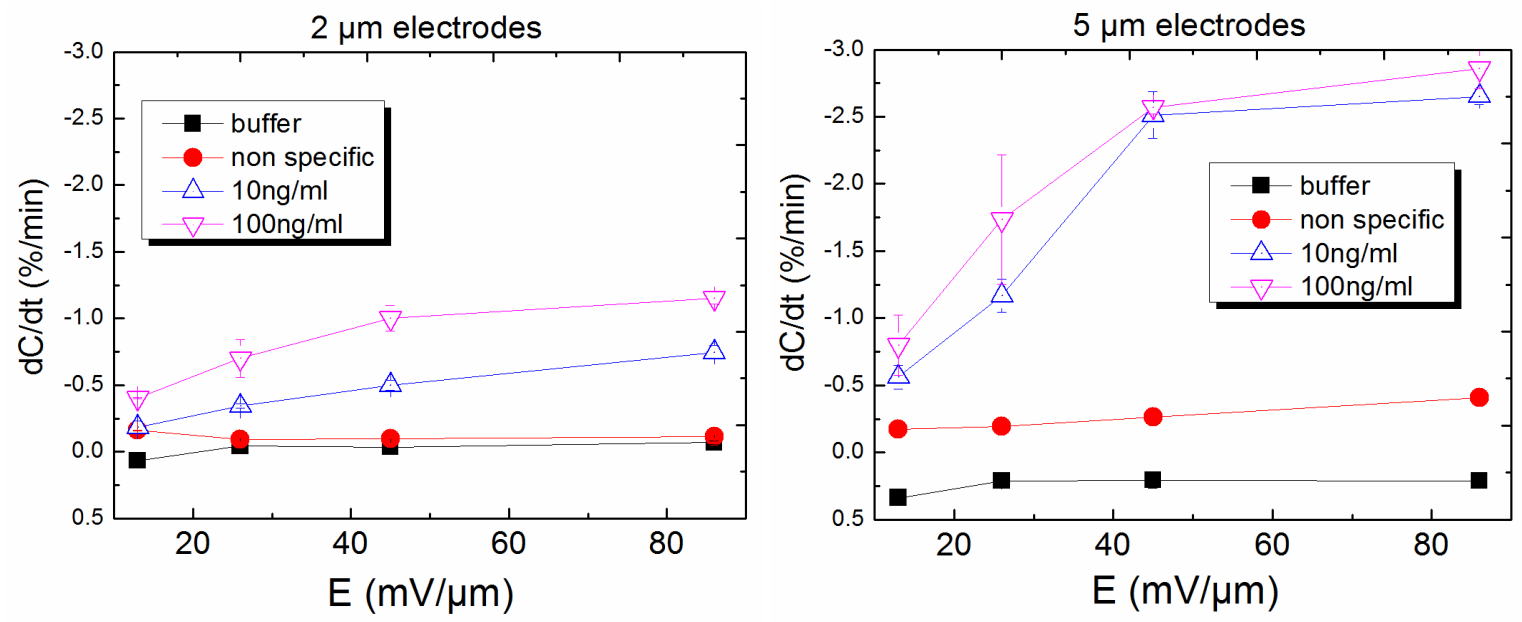

Figure.4 Binding responses of control buffer, non-specific, 10ng/mL and 100ng/mL samples under different electric field strengths. $2 \mu \mathrm{m}$ and $5 \mu \mathrm{m}$ electrodes were tested for comparison. Each test was repeated twice and the error bar indicates standard deviation.

Next, the effect of AC electric field on enhancing binding was studied further by varying AC electric field strength from $13 \mathrm{mV} / \mu \mathrm{m}$ to $86 \mathrm{mV} / \mu \mathrm{m}$. This helped us to establish a range of electric field strength for effective improvement by ACEK effects. Only the results from $2 \mu \mathrm{m}$ and $5 \mu \mathrm{m}$ electrodes to 10 and $100 \mathrm{ng} / \mathrm{mL} \mathrm{IgG}$ solutions were shown here in Fig. 4 for its clarity. Four characteristics could be noticed from Fig. 4. First, at $13 \mathrm{mV} / \mu \mathrm{m}$, the sensor responses were small for all cases, even for $100 \mathrm{ng} / \mathrm{mL}$ sample on $5 \mu \mathrm{m}$ electrodes. This indicated that this electric field strength was too low to induce sufficient ACEK effects to effectively enhance binding reactions. For $5 \mu \mathrm{m}$ electrodes, the change rates were slightly more negative than $0.30 \% /$ minute, the cut-off point for a detection. Second, the capacitance change rate increased steadily with electric field strength, indicating that binding was aided by AC electric field, which strongly supports that low AC electric field can induce usable DEP and ACET effects. Third, the difference in the responses between $2 \mu \mathrm{m}$ and $5 \mu \mathrm{m}$ electrodes widened quickly at higher electric field strength, especially for responses from 10ng/mL IgG samples, consistent with Eq. (2) of ACET force $F_{A C E T} \propto r|E|^{4}$. So it is clear that enrichment by ACET effect is more dominant in larger electrodes. Last, the rate of increase in sensor response became less when the electric field strength goes above $45 \mathrm{mV} / \mu \mathrm{m}$, especially for $5 \mu \mathrm{m}$ electrodes. So the range of AC electric field strength was estimated to be between 13 and $45 \mathrm{mV} / \mu \mathrm{m}$ for effective induction of ACEK enhancement in affinity sensing.

When the electric field strength was higher than $45 \mathrm{mV} / \mu \mathrm{m}$, ACEK effects became so strong that sufficient analyte molecules were transported close to the electrodes, and the surface binding shifted from transport-limited domain to reaction-limited domain, i.e. showing saturation of binding sites. An example is the outputs from $5 \mu \mathrm{m}$ electrodes. There was little difference 
between its response to 10 and $100 \mathrm{ng} / \mathrm{mL} \mathrm{IgG} \mathrm{samples,} \mathrm{and} \mathrm{there} \mathrm{was} \mathrm{no} \mathrm{increase} \mathrm{in} \mathrm{sensor}$ response at electric field higher than $45 \mathrm{mV} / \mu \mathrm{m}$.

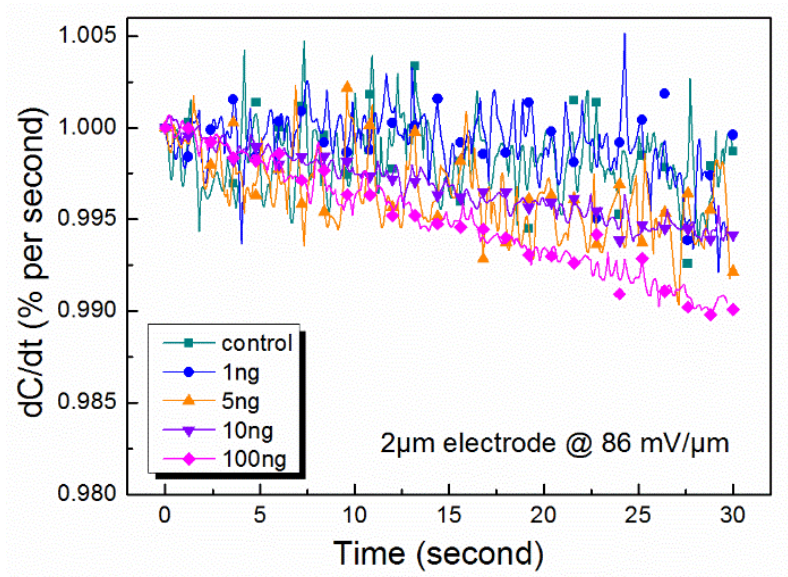

(a)

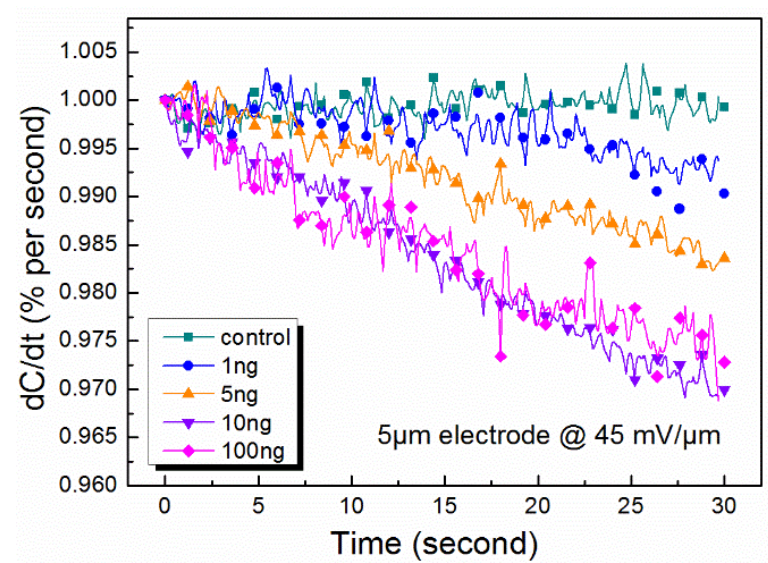

(b)

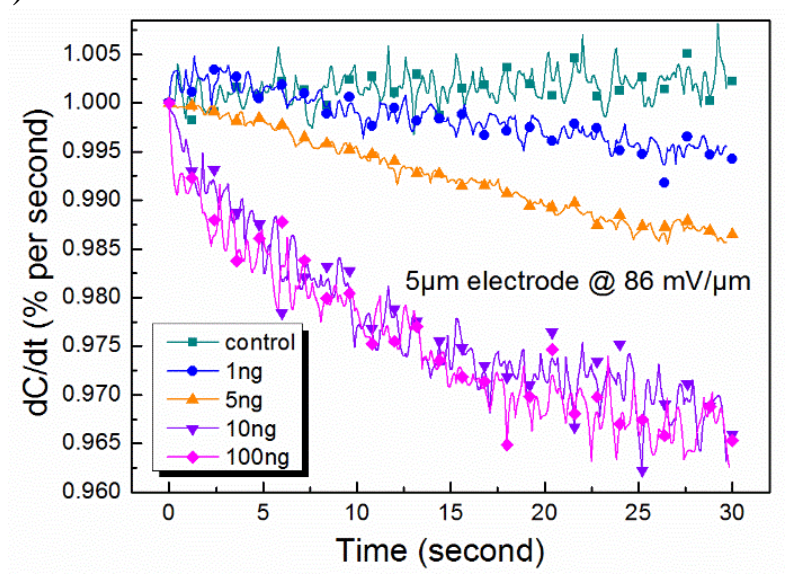

(c)

Figure. 5 Normalized capacitance changes with time. (a) $2 \mu \mathrm{m}$ electrodes measured at $86 \mathrm{mV} / \mu \mathrm{m}$;

(b) $5 \mu \mathrm{m}$ electrodes measured at $45 \mathrm{mV} / \mu \mathrm{m}$; (c) $5 \mu \mathrm{m}$ electrodes measured at $86 \mathrm{mV} / \mu \mathrm{m}$.

A comparison of the output transients between $2 \mu \mathrm{m}$ and $5 \mu \mathrm{m}$ electrodes showed that ACET flows were indeed the cause of binding saturation, as shown in Fig. 5. For $2 \mu \mathrm{m}$ electrodes at 86 $\mathrm{mV} / \mu \mathrm{m}$, as shown in Fig. 5a, the response still consistently became larger with more concentrated IgG samples. The interfacial capacitance decreased linearly with time with little indication of saturation. However, for $5 \mu \mathrm{m}$ electrodes, saturation began to show for IgG above $10 \mathrm{ng} / \mathrm{mL}$ even at $45 \mathrm{mV} / \mu \mathrm{m}$. The change rate in normalized capacitance became larger as $\mathrm{IgG}$ concentration increased from $1 \mathrm{ng} / \mathrm{mL}$ to $5 \mathrm{ng} / \mathrm{mL}, 10 \mathrm{ng} / \mathrm{mL}$. For $100 \mathrm{ng} / \mathrm{mL}$, the capacitance initially dropped quickly then became more gradual after 10 seconds, which was indicative of slowdown in binding reaction. At $86 \mathrm{mV} / \mu \mathrm{m}$, the indication of saturation kinetics became much 
more pronounced. The capacitance curves for $10 \mathrm{ng} / \mathrm{mL}$ and $100 \mathrm{ng} / \mathrm{mL}$ overlapped each other and asymptotically approached a maximum of capacitance change, characteristic of specific binding.

Table. $2 \mathrm{dC} / \mathrm{dt}(\% / \mathrm{min})$ of 1 and $5 \mathrm{ng} / \mathrm{mL}$ for every 10 seconds

\begin{tabular}{|c|c|c|c|}
\hline Conditions & $0-10 \mathrm{sec}$. & $10-20 \mathrm{sec}$. & $20-30 \mathrm{sec}$. \\
\hline $2 \mu \mathrm{m} @ 86 \mathrm{mV} / \mu \mathrm{m}(1 \mathrm{ng} / \mathrm{mL})$ & 0.40 & -0.29 & -0.82 \\
\hline $5 \mu \mathrm{m} @ 45 \mathrm{mV} / \mu \mathrm{m}(1 \mathrm{ng} / \mathrm{mL})$ & -0.04 & -0.67 & -1.53 \\
\hline $5 \mu \mathrm{m} @ 86 \mathrm{mV} / \mu \mathrm{m}(1 \mathrm{ng} / \mathrm{mL})$ & -1.13 & -0.56 & -0.66 \\
\hline $5 \mu \mathrm{m} @ 86 \mathrm{mV} / \mu \mathrm{m}(5 \mathrm{ng} / \mathrm{mL})$ & -0.90 & -1.42 & -1.37 \\
\hline
\end{tabular}

Table. 2 showed the capacitance change rate of 1 and $5 \mathrm{ng} / \mathrm{mL} \mathrm{IgG} \mathrm{sample} \mathrm{at} \mathrm{10-second} \mathrm{time}$ interval. It can be seen clearly that binding was transport-limited for $2 \mu \mathrm{m}$ electrodes or $5 \mu \mathrm{m}$ electrodes at at $45 \mathrm{mV} / \mu \mathrm{m}$, and reaction-limited for $5 \mu \mathrm{m}$ electrodes at electric field higher than at $45 \mathrm{mV} / \mu \mathrm{m}$. In the first 10 seconds, $2 \mu \mathrm{m}$ electrode showed a capacitance change rate of $0.40 \% /$ minute. As time went by, the change rate became more negative from $-0.29 \% / \mathrm{min}$ in the $10-20$ seconds to $-0.82 \% / \mathrm{min}$ in the $20-30$ seconds, which indicated increasing binding rate as a result of biomolecules being gradually transported to the vicinity of electrode surface. Similar situation was shown for $5 \mu \mathrm{m}$ electrodes at $45 \mathrm{mV} / \mu \mathrm{m}$. In comparison, it was a different scenario for $5 \mu \mathrm{m}$ electrodes at $86 \mathrm{mV} / \mu \mathrm{m}$. At $1 \mathrm{ng} / \mathrm{mL}$, its change rate was $-1.13 \% / \mathrm{min}$ in the first 10 seconds and slowed down in subsequent time periods, indicating a faster transport of analytes at $86 \mathrm{mV} / \mu \mathrm{m}$ than at $45 \mathrm{mV} / \mu \mathrm{m}$. At $5 \mathrm{ng} / \mathrm{mL}$, the $\mathrm{C}_{\text {int }}$ change rate was fairly constant, probably because there were plenty of analytes for binding. Based on Table. 2, larger electrodes were clearly better at enriching analytes. Both the much larger response and its transient behavior of 5 $\mu \mathrm{m}$ electrodes indicated that $5 \mu \mathrm{m}$ electrodes can induce much stronger ACEK effect, mostly ACET effect, to enhance detection of dilute analytes.

From a physics viewpoint, electric field of $2 \mu \mathrm{m}$ or smaller electrodes was concentrated around the electrode corners and between the electrodes, while electric field of $5 \mu \mathrm{m}$ electrodes could reach further into the fluids. ACET effect on fluid, as a body force, needs some distance away from solid phase to effectively generate convection [52]. Otherwise, the friction from the solid surface will be overwhelming (no slip boundary condition). Consequently, $5 \mu \mathrm{m}$ electrodes was able to generate much stronger ACET convections than $2 \mu \mathrm{m}$ electrodes, which in turn provided a much larger binding response.

\section{b) Small molecule detection}

Next, detection of small molecules was conducted to further demonstrate ACET enrichment, as the relative importance of DEP and ACET forces shifts in favor of ACET effect with smaller molecules. BPA was used as a model for small molecule detection. BPA is reported as an 
endocrine-disrupting chemical and detection of BPA level within the environment as well as human bodily fluids is of crucial importance [53, 54]. In recent studies, the LoD of BPA is reported to be $0.11 \mathrm{ng} / \mathrm{mL}$ by high performance liquid chromatography [55] and $3.1 \mathrm{ng} / \mathrm{mL}$ by ELISA [56]. Compared with IgG antibody which has a molecular weight of $150 \mathrm{kDa}$, the molecular weight of BPA is only 228 Da. As can be seen from Eqs.1 and 2, DEP force is dependent on the particle size while ACET effect is not. As a result, DEP enrichment will be much weaker for BPA than for IgG. So the use of ACET effect will be more important to small molecule detection. Both SAW and $5 \mu \mathrm{m}$ electrodes were used for comparison. To contrast the effect of electrode characteristic length, the applied electric field strength is $212 \mathrm{mV} / \mu \mathrm{m}$ on SAW electrodes and $45 \mathrm{mV} / \mu \mathrm{m}$ on $5 \mu \mathrm{m}$ electrodes.

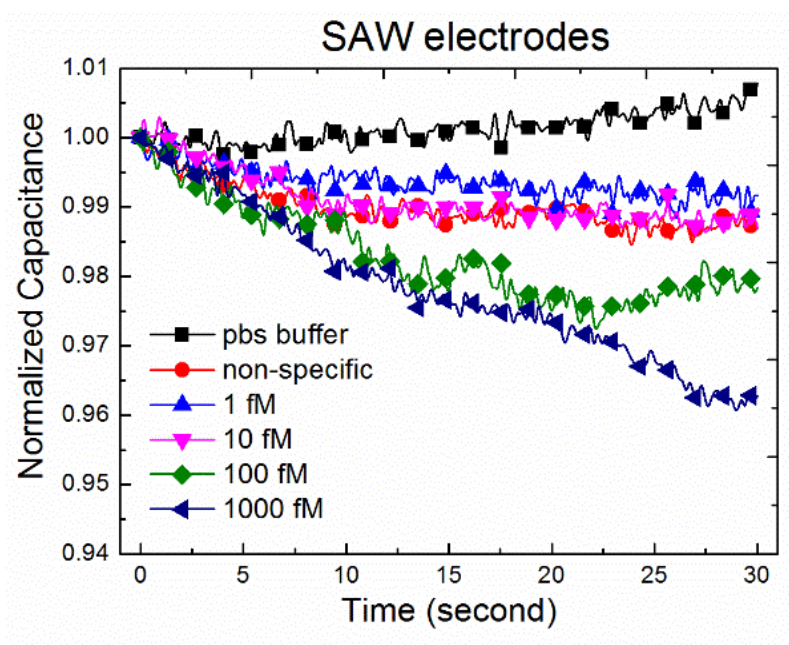

(a)

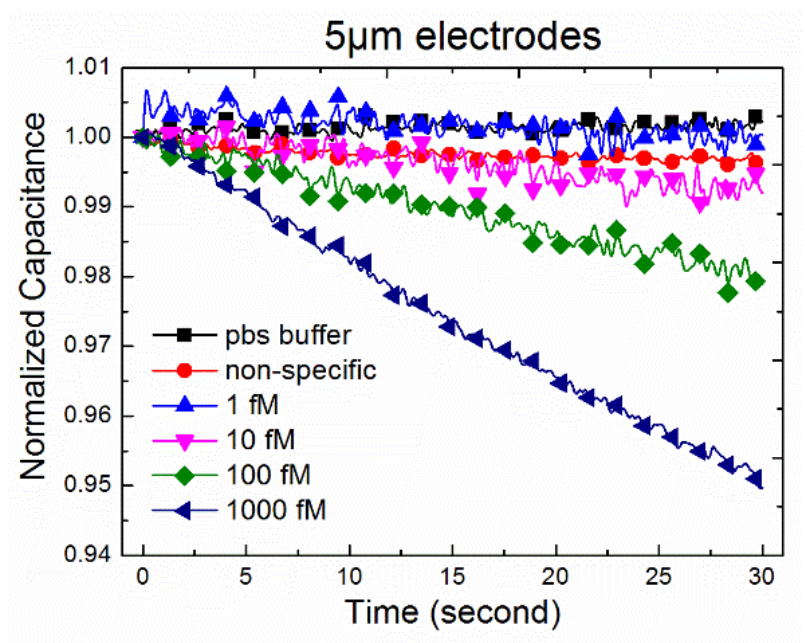

(b)

Figure.6 BPA detection responses from (a) SAW electrodes and (b) $5 \mu \mathrm{m}$ electrodes. Each curve is real time change of normalized interfacial capacitance for different samples. Buffer solution

is $0.1 \mathrm{x}$ PBS on functionalized electrodes, non-specific tests are $1000 \mathrm{fM}$ BPA on dummy electrodes; 1-1000 fM are BPA samples on functionalized electrodes. The capacitance change was tracked for 30 seconds.

The results of BPA detection was shown in Fig. 6. In Fig. 6, the rate of decrease in capacitance steadily became larger with more concentrated samples on both electrodes. The responses exhibited a logarithmic dependence on BPA concentration ranging from 1 to $100 \mathrm{fM}$ (i.e. $0.28-28 \mathrm{fg} / \mathrm{mL}$ ) for both electrodes. Baseline responses were obtained from measuring buffer solution, which were also around zero, $0.01 \pm 0.02 \% / \mathrm{min}$ on $5 \mu \mathrm{m}$ electrodes and $0.16 \pm$ $0.15 \% / \mathrm{min}$ on SAW electrodes, showing no appreciable binding. For non-specific binding of $100 \mathrm{fM}$ BPA on dummy sensors, the curve went down slightly, indicating low level of nonspecific binding (red line), with a slope comparable to $1 \mathrm{fM}$ specific binding. 
For SAW electrodes, signs of BPA depletion could be seen, as the curves gradually became flatter after a few seconds of tests. The saturation became less obvious at higher BPA levels. For $5 \mu \mathrm{m}$ electrodes, there was little indication of saturation, since the capacitance went down almost linearly with time. Even though much higher electric field strength was applied on SAW electrode, larger response was achieved with $5 \mu \mathrm{m}$ electrodes, clearly due to the strong effect of ACET convection.

To reduce the effect of analyte depletion on SAW electrodes, the sensor response was calculated using the first 20 second data. LOD was determined to be $10 \mathrm{fM}(2.8 \mathrm{fg} / \mathrm{mL})$ for $5 \mu \mathrm{m}$ electrode and $100 \mathrm{fM}(28 \mathrm{fg} / \mathrm{mL})$ for SAW electrodes. As shown in Figure.7, $5 \mu \mathrm{m}$ electrode produced a change rate of $-0.50 \pm 0.11 \% /$ min for $10 \mathrm{fM}$ BPA, which can easily be distinguished from the baseline. SAW electrodes yielded a change rate of $-0.36 \pm 0.07 \% / \mathrm{min}$ for $100 \mathrm{fM}$ BPA. Similarly, $5 \mu \mathrm{m}$ electrodes were more sensitive than SAW electrodes. The detection sensitivity of BPA on $5 \mu \mathrm{m}$ electrodes is calculated to be $-0.40 \pm 0.08 \% / \log$ (fM), much higher than that on SAW electrodes, which is $-0.09 \pm 0.04 \% / \log$ (fM). Responses from non-specific binding were minimal on both electrodes for all BPA concentrations tested. $100 \mathrm{fM}$ BPA caused a change rate of $-0.10 \pm 0.02 \% / \mathrm{min}$ for $5 \mu \mathrm{m}$ electrodes, and $0.01 \pm 0.11 \% / \mathrm{min}$ for SAW electrodes, showing good specificity. Cross-reactivity of the BPA sensor was also tested against $1 \mathrm{pM}$ and $10 \mathrm{pM}$ BPS, a molecule structurally similar to BPA. The response was determined to be non-reactive (please see the details and data in Supplementary Material section).

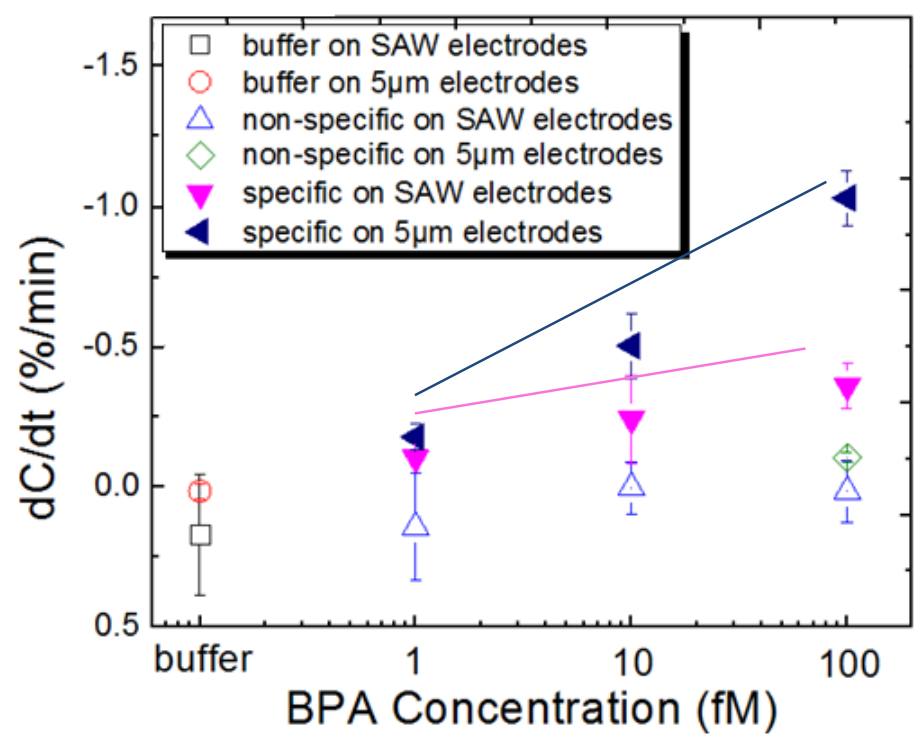

Figure.7 BPA detection results on both electrodes. Buffer tests are 0.1x PBS on functionalized electrodes; non-specific tests are 1-100 fM BPA on dummy electrodes; specific tests use 1-100 fM BPA samples on functionalized electrodes. Each data point was the average of three repeated tests and the error bar indicates standard deviation. 


\section{Conclusions}

Here we presented a low voltage ACEK capacitive affinity sensing platform capable of simple, rapid yet sensitive detection of specific molecules in biological samples. As a follow up to our previous work, the effects of low voltage AC electric field on affinity binding were studied further with respect to different electrode geometries. For protein sensors, detection sensitivity and limit of detection were improved over our prior reports when larger electrodes with a characteristic length of $5 \mu \mathrm{m}$ were used. This was attributed to the stronger ability of larger electrodes to induce ACET microflows, hence higher sensor response. The benefits from ACET effect became more important for low abundance analyte since longer range of enrichment by ACET flows was needed, and for small molecule analyte since ACET enrichment was independent of analyte size. In addition, the study found that a characteristic electric field strength of $45 \mathrm{mV} / \mu \mathrm{m}$ was close to be the optimal AC field for ACEK capacitive sensing,. In this work, sensitive, quantitative detection was demonstrated for protein (bovine $\operatorname{IgG}$ ) and small molecules (BPA). LODs of $1 \mathrm{ng} / \mathrm{mL}$ IgG and $10 \mathrm{fM}$ BPA were achieved, which were lower than most existing technologies. Overall, ACEK capacitive sensing method possesses great potential as becoming a real-world point-of-care diagnostics tool in the future.

\section{Acknowledgement}

Microfabrication of this research was conducted at the Center for Nanophase Materials Sciences, which is sponsored at Oak Ridge National Laboratory by the Scientific User Facilities Division, US Department of Energy. BPA detection was supported by the University of Tennessee Center for Wildlife Health Organized Research Unit. X. Lin acknowledges the financial support for his BPA detection work at the University at Tennessee from China Scholarship Council. His work was also supported by National Natural Science Foundation of China (Project no. 6137 7001), the Fundamental Research Funds for the Central Universities (Project no.CDJZR12 125501 and Project no. 10611201312011). 


\section{References}

[1] B. Pejcic, R. De Marco, G. Parkinson, The role of biosensors in the detection of emerging infectious diseases, Analyst, 131(2006) 1079-90.

[2] J.S. Daniels, N. Pourmand, Label - free impedance biosensors: Opportunities and challenges, Electroanalysis, 19(2007) 1239-57.

[3] N. Karimian, A.P. Turner, A. Tiwari, Electrochemical evaluation of troponin T imprinted polymer receptor, Biosensors and Bioelectronics, 59(2014) 160-5.

[4] O. Parlak, A. Tiwari, A.P. Turner, A. Tiwari, Template-directed hierarchical self-assembly of graphene based hybrid structure for electrochemical biosensing, Biosensors and Bioelectronics, 49(2013) 53-62.

[5] Y. Wan, Y. Su, X. Zhu, G. Liu, C. Fan, Development of electrochemical immunosensors towards point of care diagnostics, Biosensors and Bioelectronics, 47(2013) 1-11.

[6] Y. Xu, E. Wang, Electrochemical biosensors based on magnetic micro/nano particles, Electrochimica Acta, 84(2012) 62-73.

[7] J.C. Vidal, L. Bonel, A. Ezquerra, S. Hernández, J.R. Bertolín, C. Cubel, et al., Electrochemical affinity biosensors for detection of mycotoxins: A review, Biosensors and Bioelectronics, 49(2013) 146-58.

[8] M.I. Prodromidis, Impedimetric immunosensors-A review, Electrochimica Acta, 55(2010) 4227-33.

[9] D.Z. Pan, T. Viswanathan, Integrated Impedance Spectroscopy Biosensors.

[10] D.A. Hall, J. Ptacek, M. Snyder, Protein microarray technology, Mechanisms of ageing and development, 128(2007) 161-7.

[11] O. Stoevesandt, M.J. Taussig, M. He, Protein microarrays: high-throughput tools for proteomics, (2009).

[12] M. Cretich, F. Damin, M. Chiari, Protein microarray technology: how far off is routine diagnostics?, Analyst, 139(2014) 528-42.

[13] S. Loizou, J. McCrea, A. Rudge, R. Reynolds, C. Boyle, E. Harris, Measurement of anti-cardiolipin antibodies by an enzyme-linked immunosorbent assay (ELISA): standardization and quantitation of results, Clinical and experimental immunology, 62(1985) 738.

[14] P.E. Sheehan, L.J. Whitman, Detection limits for nanoscale biosensors, Nano letters, 5(2005) 803-7.

[15] Z.J. Sahab, S.M. Semaan, Q.-X.A. Sang, Methodology and applications of disease biomarker identification in human serum, Biomarker Insights, 2(2007) 21.

[16] J. Wang, Electrochemical biosensors: towards point-of-care cancer diagnostics, Biosensors and Bioelectronics, 21(2006) 1887-92.

[17] D.S. Smith, S.A. Eremin, Fluorescence polarization immunoassays and related methods for simple, high-throughput screening of small molecules, Analytical and bioanalytical chemistry, 391(2008) 1499507.

[18] G. Lai, J. Wu, H. Ju, F. Yan, Streptavidin - Functionalized Silver - Nanoparticle - Enriched Carbon Nanotube Tag for Ultrasensitive Multiplexed Detection of Tumor Markers, Advanced Functional Materials, 21(2011) 2938-43.

[19] P. Du, H. Li, Z. Mei, S. Liu, Electrochemical DNA biosensor for the detection of DNA hybridization with the amplification of Au nanoparticles and CdS nanoparticles, Bioelectrochemistry, 75(2009) 37-43. [20] S.H. Lee, D. van Noort, J.Y. Lee, B.-T. Zhang, T.H. Park, Effective mixing in a microfluidic chip using magnetic particles, Lab on a Chip, 9(2009) 479-82.

[21] M. Sigurdson, D. Wang, C.D. Meinhart, Electrothermal stirring for heterogeneous immunoassays, Lab on a Chip, 5(2005) 1366-73.

[22] J. Wu, Interactions of electrical fields with fluids: laboratory-on-a-chip applications, IET Nanobiotechnology, 2(2008) 14-27.

[23] H. Morgan, N.G. Green, AC electrokinetics: colloids and nanoparticles: Research Studies Press; 2003. 
[24] J. Wu, M. Lian, K. Yang, Micropumping of biofluids by alternating current electrothermal effects, Applied physics letters, 90(2007) 234103--3.

[25] M. Lian, N. Islam, J. Wu, AC electrothermal manipulation of conductive fluids and particles for labchip applications, IET Nanobiotechnology, 1(2007) 36-43.

[26] J. Wu, Y. Ben, H.-C. Chang, Particle detection by electrical impedance spectroscopy with asymmetric-polarization AC electroosmotic trapping, Microfluidics and Nanofluidics, 1(2005) 161-7.

[27] X. Liu, K. Yang, A. Wadhwa, S. Eda, S. Li, J. Wu, Development of an AC electrokinetics-based immunoassay system for on-site serodiagnosis of infectious diseases, Sensors and Actuators A: Physical, 171(2011) 406-13.

[28] R. Hamada, H. Takayama, Y. Shonishi, L. Mao, M. Nakano, J. Suehiro, A rapid bacteria detection technique utilizing impedance measurement combined with positive and negative dielectrophoresis, Sensors and Actuators B: Chemical, 181(2013) 439-45.

[29] J. Wu, N. Islam, A simple method to integrate in situ nano-particle focusing with cantilever detection, Sensors Journal, IEEE, 7(2007) 957-8.

[30] Y. Zaikov, A. Khramov, V. Kovrov, V. Kryukovsky, A. Apisarov, O. Tkacheva, et al., Electrolysis of aluminum in the low melting electrolytes based on potassium cryolite, LIGHT METALS-WARRENDALEPROCEEDINGS-, TMS2008, p. 505.

[31] Y. Xiong, Y. Liu, Biological control of microbial attachment: a promising alternative for mitigating membrane biofouling, Applied microbiology and biotechnology, 86(2010) 825-37.

[32] M.J. Shiddiky, R. Vaidyanathan, S. Rauf, Z. Tay, M. Trau, Molecular Nanoshearing: An Innovative Approach to Shear off Molecules with AC-Induced Nanoscopic Fluid Flow, Scientific reports, 4(2014). [33] H. Cui, S. Li, Q. Yuan, A. Wadhwa, S. Eda, M. Chambers, et al., An AC electrokinetic impedance immunosensor for rapid detection of tuberculosis, Analyst, 138(2013) 7188-96.

[34] S. Li, H. Cui, Q. Yuan, J. Wu, A. Wadhwa, S. Eda, et al., AC electrokinetics-enhanced capacitive immunosensor for point-of-care serodiagnosis of infectious diseases, Biosensors and Bioelectronics, 51(2014) 437-43.

[35] S. Li, Y. Ren, H. Cui, Q. Yuan, J. Wu, S. Eda, et al., Alternating current electrokinetics enhanced in situ capacitive immunoassay, Electrophoresis, 36(2015) 471-4.

[36] S. Eda, J.P. Bannantine, W. Waters, Y. Mori, R.H. Whitlock, M.C. Scott, et al., A highly sensitive and subspecies-specific surface antigen enzyme-linked immunosorbent assay for diagnosis of Johne's disease, Clinical and vaccine immunology, 13(2006) 837-44.

[37] H. Cui, C. Cheng, J. Wu, S. Eda, Rapid detection of progesterone by commercially available microelectrode chips, Sensors, 2013 IEEE, IEEE2013, pp. 1-4.

[38] R. Hart, R. Lec, H. Noh, Enhancement of heterogeneous immunoassays using AC electroosmosis, Sensors and Actuators B: Chemical, 147(2010) 366-75.

[39] J. Wu, Biased AC electro-osmosis for on-chip bioparticle processing, Nanotechnology, IEEE Transactions on, 5(2006) 84-9.

[40] R. Zhang, C. Dalton, G.A. Jullien, Two-phase AC electrothermal fluidic pumping in a coplanar asymmetric electrode array, Microfluidics and nanofluidics, 10(2011) 521-9.

[41] A. Castellanos, A. Ramos, A. Gonzalez, N.G. Green, H. Morgan, Electrohydrodynamics and dielectrophoresis in microsystems: scaling laws, Journal of Physics D: Applied Physics, 36(2003) 2584.

[42] R. Reverberi, L. Reverberi, Factors affecting the antigen-antibody reaction, Blood Transfusion, 5(2007) 227.

[43] N.G. Green, A. Ramos, A. Gonzalez, H. Morgan, A. Castellanos, Fluid flow induced by nonuniform ac electric fields in electrolytes on microelectrodes. I. Experimental measurements, Physical review $E$, 61(2000) 4011. 
[44] C.-K. Yang, J.-S. Chang, S.D. Chao, K.-C. Wu, Two dimensional simulation on immunoassay for a biosensor with applying electrothermal effect, Applied Physics Letters, 91(2007) 113904.

[45] H.C. Feldman, M. Sigurdson, C.D. Meinhart, AC electrothermal enhancement of heterogeneous assays in microfluidics, Lab on a Chip, 7(2007) 1553-9.

[46] Q. Yuan, K. Yang, J. Wu, Optimization of planar interdigitated microelectrode array for biofluid transport by AC electrothermal effect, Microfluidics and nanofluidics, 16(2014) 167-78.

[47] K. Yang, J. Wu, Numerical study of in situ preconcentration for rapid and sensitive nanoparticle detection, Biomicrofluidics, 4(2010) 034106.

[48] Z. Mei, H. Chu, W. Chen, F. Xue, J. Liu, H. Xu, et al., Ultrasensitive one-step rapid visual detection of bisphenol A in water samples by label-free aptasensor, Biosensors and Bioelectronics, 39(2013) 26-30.

[49] H. Cui, J. Wu, S. Eda, J. Chen, W. Chen, L. Zheng, Rapid capacitive detection of femtomolar levels of bisphenol A using an aptamer-modified disposable microelectrode array, Microchimica Acta, 182(2015)

2361-7.

[50] S. Li, Q. Yuan, B. Morshed, C. Ke, J. Wu and H. Jiang, "Dielectrophoretic Response of DNA and Fluorophore in Physiological Solution by Impedimetric Characterization," Biosensors \& Bioelectronics, Vol. 41, pp. 649-655, 2013.

[51] G. Nahler, G. Nahler, Pearson correlation coefficient, Dictionary of Pharmaceutical Medicine, (2009) 132.

[52] S. Loire, P. Kauffmann, I. Mezić, C. Meinhart, A theoretical and experimental study of ac electrothermal flows, Journal of Physics D: Applied Physics, 45(2012) 185301.

[53] C.A. Staples, P.B. Dome, G.M. Klecka, S.T. Oblock, L.R. Harris, A review of the environmental fate, effects, and exposures of bisphenol A, Chemosphere, 36(1998) 2149-73.

[54] F. Xue, J. Wu, H. Chu, Z. Mei, Y. Ye, J. Liu, et al., Electrochemical aptasensor for the determination of bisphenol A in drinking water, Microchimica Acta, 180(2013) 109-15.

[55] R. Braunrath, D. Podlipna, S. Padlesak, M. Cichna-Markl, Determination of bisphenol A in canned foods by immunoaffinity chromatography, HPLC, and fluorescence detection, Journal of agricultural and food chemistry, 53(2005) 8911-7.

[56] A. Kim, C.-R. Li, C.-F. Jin, K.W. Lee, S.-H. Lee, K.-J. Shon, et al., A sensitive and reliable quantification method for bisphenol A based on modified competitive ELISA method, Chemosphere, 68(2007) 1204-9. 